\title{
The Effect of Senescence of Endothelial Progenitor Cells on In-stent Restenosis in Patients Undergoing Coronary Stenting
}

\author{
Yoshiki Matsuo ${ }^{1}$, Toshio Imanishi ${ }^{1}$, Yasushi Hayashi ${ }^{2}$, Yoshiaki Tomobuchi ${ }^{2}$, \\ Takashi Kubo ${ }^{1}$, Takuzo Hano ${ }^{1}$ and Takashi Akasaka ${ }^{1}$
}

\begin{abstract}
Objective Restenosis after stent implantation is caused by endothelial cell damage and subsequent neointimal formation. The objective of this study is to elucidate the relevance of endothelial progenitor cells (EPCs) in the development of in-stent restenosis in patients undergoing stent implantation.

Patients or Materials The subjects were 46 patients who underwent coronary stenting. Blood samples were collected at the time of follow-up coronary angiography after coronary stenting. EPCs were isolated from blood samples and cultured. Their phenotypes were confirmed by uptake of acetylated low-density lipoprotein and binding of fluorescein isothiocyanate-labeled Ulex europaeus agglutinin 1 lectin. The number of colony-forming units (CFUs) and the senescent cells, determined by acidic $\beta$-galactosidase staining, was counted. Angiogenic growth factors secreted by EPCs, such as vascular endothelial growth factor (VEGF), basic fibroblast growth factor (b-FGF), hepatocyte growth factor (HGF), and macrophage chemoattractant protein (MCP-1) from the culture medium were also measured by enzyme-linked immunosorbent assay.

Results Patients with in-stent restenosis (defined as $>40 \%$ stenosis, $n=16$ ) had a decreased number of CFUs $(\mathrm{p}<0.05)$, and increased senescent cells $(\mathrm{p}<0.05)$, compared to patients without restenosis $(n=30)$. There was no significant difference of angiogenic growth factors (VEGF, HGF, b-FGF, and MCP-1) secreted by EPCs between the two groups. On multivariate analysis, an increased number of senescent EPCs was the independent factor associated with in-stent restenosis (OR 1.10, 95\% CI 1.01 to 1.20 ).
\end{abstract}

Conclusion These data suggested that EPCs might be involved in the development of in-stent restenosis.

Key words: endothelial progenitor cell, in-stent restenosis, reendothelialization

(DOI: 10.2169/internalmedicine.45.1663)

\section{Introduction}

Percutaneous coronary intervention (PCI) with stenting has become an effective and widespread treatment modality for patients with coronary artery disease (CAD), but in-stent restenosis (ISR) remains a recognized clinical problem. ISR occurs after PCI with bare-metal stent implantation in $10 \%$ to $40 \%$ of cases at 6 months, depending on various clinical, angiographic, and procedural features. It is evident that neointimal proliferation is the underlying cause of ISR (1).
Recent studies have identified endothelial progenitor cells (EPCs) that can be isolated from circulating mononuclear cells (2), and risk factors for atherosclerosis are associated with reduced levels of circulating EPCs (3).

A rapid regeneration of the endothelial monolayer may prevent restenosis development by endothelial synthesis of antiproliferative mediators. In the past, the regeneration of injured endothelium has been attributed to the migration and proliferation of neighboring endothelial cells. A recent study, however, indicated that transfusion of EPCs results in reduced intimal thickening due to accelerated reendothelializa-

\footnotetext{
${ }^{1}$ Department of Cardiovascular Medicine, Wakayama Medical University, Wakayama and ${ }^{2}$ Department of Critical Care Medicine, Wakayama Medical University, Wakayama

Received for publication November 24, 2005; Accepted for publication March 8, 2006

Correspondence to Toshio Imanishi, Department of Cardiovascular Medicine, Wakayama Medical University, 811-1 Kimiidera, Wakayama 6418509
} 


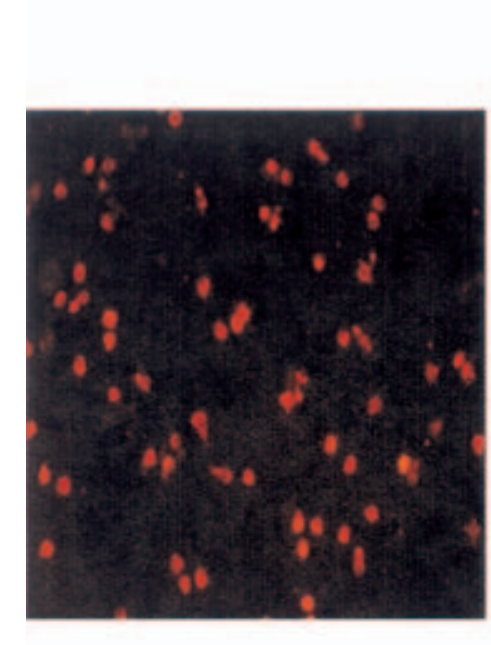

DiLDL

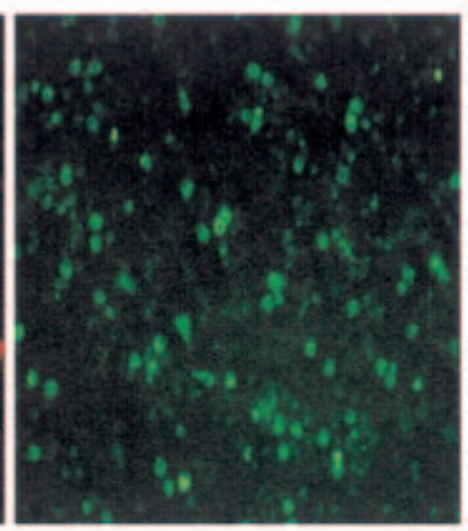

UEA-1 lectin

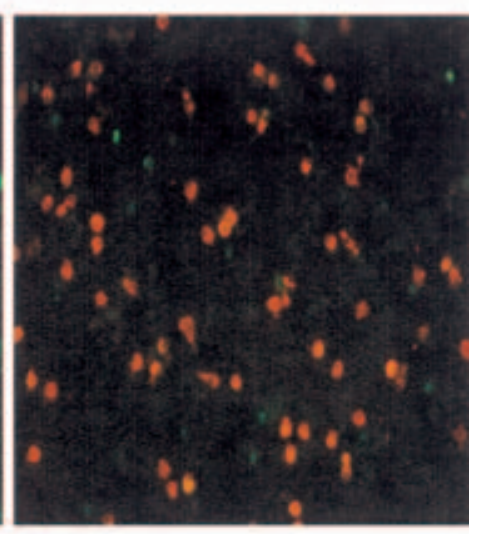

Dual staining

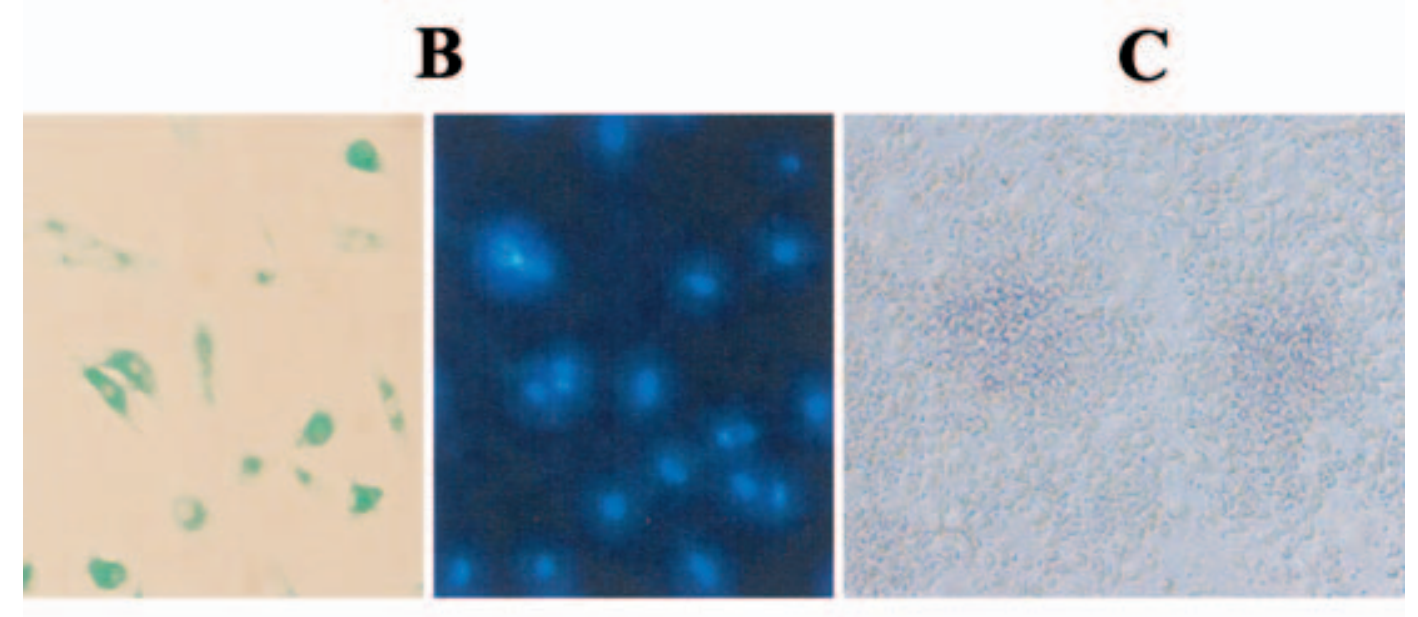

$\beta-$ Gal DAPI $\quad$ EPC Colonies

Figure 1. A, Characterization of circulating EPCs. Expression of endothelial markers, Di-LDL (left), lectin (middle), and dual staining (right). B, Representative $\beta$-galactosidase activity ( $\beta$ - gal) (left), and DAPI staining (right). C, Representative colony-forming units are shown at 7 days after cultivation.

tion in a mouse model (4).

Taken together, these studies have led to the hypothesis that individual characteristics of circulating EPCs would affect the reendothelialization of injured arteries after stenting and subsequent in-stent neointimal hyperplasia.

The aim of this study was to evaluate the relationships between colony-forming capacity, senescence of EPCs, angiogenic growth factors secreted from EPCs, and ISR 6 months after successful coronary stenting in patients with CAD.

\section{Materials and Methods}

\section{Patient population}

This study included 46 consecutive patients who had successful PCI of de novo lesions under emergent or elective situations in our institution. They were scheduled for followup coronary angiography after 6 months. The study protocol had been approved by the university ethics committee, and written informed consent was obtained. Subjects were solicited through the Wakayama Medical University. Subjects were excluded from this study if they had chronic renal failure (serum creatinine; $>3 \mathrm{mg} / \mathrm{dl}$ ), or had concomitant inflammatory or malignant disease. 
Table 1. Clinical and Angiographical Features in Patients with and without Instent Restenosis

\begin{tabular}{|c|c|c|c|}
\hline & $\begin{array}{c}\text { Patients With } \\
\text { ISR } \\
(\mathrm{n}=16)\end{array}$ & $\begin{array}{c}\text { Patients Without } \\
\text { ISR } \\
(\mathrm{n}=30)\end{array}$ & $\mathbf{P}$ \\
\hline Age,$y$ & $69.2 \pm 9.4$ & $67.4 \pm 8.4$ & 0.31 \\
\hline Male, $\mathbf{n}(\%)$ & $14(87.5)$ & $26(86.7)$ & 0.47 \\
\hline Acute coronary syndrome, $n(\%)$ & $8(50)$ & $14(46.7)$ & 0.42 \\
\hline \multicolumn{4}{|l|}{ Coronary risk factors } \\
\hline Smoking, $\mathrm{a}(\%)$ & $13(81.2)$ & $22(73.3)$ & 0.27 \\
\hline Hyperteasion, n(\%) & $14(87.5)$ & $22(73.3)$ & 0.12 \\
\hline Diabetes Mellitus, n(\%) & $5(31.3)$ & 7 (23.3) & 0.29 \\
\hline Hyperlipidemia, n(\%) & $12(75,0)$ & $19(63.3)$ & 0.21 \\
\hline Family history, n(\%) & $4(25,0)$ & $10(33.3)$ & 0.28 \\
\hline \multicolumn{4}{|l|}{ Medications } \\
\hline Asprin, $n(\%)$ & $16(100.0)$ & $30(100.0)$ & 1.00 \\
\hline B-bloker, $\mathrm{n}(\%)$ & $7(43.8)$ & $19(6.3)$ & 0.11 \\
\hline ACEIs/ARBs, a(\%) & $13(81.2)$ & $19(6.3 .3)$ & $0.04^{*}$ \\
\hline Statins, $\mathbf{n}(\%)$ & $10(62.5)$ & $19(6.3 .3)$ & 0.48 \\
\hline $\mathrm{Ca}$ antagonist, $\mathbf{n}(\%)$ & $4(25.0)$ & $9(30.0)$ & 0.2 .3 \\
\hline Nitrates, $\mathbf{n}(\%)$ & $7(43.8)$ & $9(30.0)$ & 0.19 \\
\hline Nicorandil, $n(\%)$ & $3(18.8)$ & $3(10.0)$ & 0.23 \\
\hline Lesion type B2/C, n(\%) & $12(75.0)$ & $19(6.3 .3)$ & 0.26 \\
\hline \multicolumn{4}{|l|}{ Stents } \\
\hline Total number ( $\mathrm{a}$ /patient) & $1.1 \pm 0.5$ & $1.1 \pm 0.3$ & 0.43 \\
\hline Stent diameter (mm) & $3.2 \pm 0.4$ & $3.4 \pm 0.4$ & $0.01^{*}$ \\
\hline Total stent length (mm) & $16.3 \pm 2.5$ & $17.0 \pm 4.0$ & 0.25 \\
\hline Maximal pressure (atm) & $15.9 \pm 3.4$ & $16.5 \pm 3.7$ & 0.28 \\
\hline
\end{tabular}

Data are expressed as mean \pm SD or number (\%) of patients, ACEIs indicates angiotensin-converting enryme inhibitors; ARBs, angiotensin receptor blockers; EPCs, endothelial progenitor cells; ISR, in-stent restenosis. "p 00.05 is considered to be significant.

\section{Coronary angiography}

Six months after coronary stenting, coronary angiography was performed in multiple orthogonal projections using the Judkins technique after intravenous bolus administration of 2000-U of heparin. Quantitative coronary angiography analysis was performed using a validated automated edgedetection program. Minimal lumen diameter, length of stenosis, and percentage diameter stenosis were measured. The presence of ISR was defined as $>40 \%$ stenosis.

\section{Definition of risk factors for CAD}

To determine the overall risk factor load of an individual subject, a risk factor score including age >65 years, male sex, hypertension, diabetes mellitus, smoking, positive family history of CAD, and hypercholesterolemia was calculated according to Vita et al (5).

\section{Isolation and characterization of EPCs}

The peripheral blood was obtained just before follow-up coronary angiography. The peripheral blood mononuclear cell fraction (PB-MNCs) was isolated by density-gradient centrifugation with Histopaque-1077 (Sigma Chemical Co., St. Louis, MO, USA) and washed three times in Dulbecco's phosphate-buffered saline (D-PBS) (GIBCO). After resuspension in endothelial cell basal medium-2 (EBM-2) (Clonetics, Walkersville, MD, USA) containing 5\% fetal bovine serum, $0.1 \%$ human VEGF-1, $0.4 \%$ human fibroblast growth factor-2 (FGF-2), $0.1 \%$ insulin-like growth factor (IGF-1), 0.1\% human epidermal growth factor (hEGF), and $0.1 \%$ ascorbic acid, PB-MNCs were placed on fibronectincoated tissue culture flasks and cultured at $37^{\circ} \mathrm{C}$ for four days. Adherent cells were incubated with 1,1'-dioctadecyl3,3,3', $3^{\prime}$-tetramethylindo-carbocyanine-labeled acethylated low-density lipoprotein (DiLDL) for one hour and fluorescein isothiocyanate (FITC)-labeled Ulex europaeus agglutinin 1 (UEA-1, Sigma Chemical Co.) for one hour. Doublestained cells were identified as EPCs (Fig. 1A). Staining of nuclei with 4',6-diamino-phenylindole (DAPI) $(0.2 \mu \mathrm{g} / \mathrm{ml}$ in $10 \mathrm{nmol} / \mathrm{l}$ Tris-HCl, $\mathrm{pH} 7.0,10 \mathrm{nmol} / \mathrm{l}$ EDTA, $100 \mathrm{nmol} / \mathrm{l}$ $\mathrm{NaCl}$ ) for $10 \mathrm{~min}$ verified that nearly all adherent cells (> 95\%) were stained positive for both DiLDL and ulex-lectin.

\section{Acidic $\beta$-galactosidase staining}

For the measurement of cellular senescence, cultures of EPCs were maintained for seven days. Senescenceassociated $\beta$-galactosidase activity was measured as previously described (6). Cells were counterstained with 4', 6diamidino-phenylindole (DAPI; $0.2 \mu \mathrm{g} / \mathrm{ml}$ in $10 \mathrm{mmol} / \mathrm{l}$ Tris-HCl, pH 7.0, $10 \mathrm{mmol} / \mathrm{l}$ EDTA, $100 \mathrm{mmol} / \mathrm{l} \mathrm{NaCl}$ ) for 10 minutes to count the total cell number. Only cells with a distinctly blue cytoplasm, indicating $\beta$-galactosidase activity, were counted (Fig. 1B). The percentage of $\beta$-galactosidasepositive cells for DAPI-positive cells was determined by counting five random fields under microscopy $(\times 20)$.

\section{Colony-forming units and growth factor secre- tion}

To assess EPC colony formation and growth factor secretion, adherent cells were detached and $1 \times 10^{6}$ cells were subsequently cultured on fibronection-coated plates with $1 \mathrm{ml}$ of basal medium EBM-2 on day 4 for 72 hours. Plates were studied under phase-contrast microscopy, and colonies (Fig. 1C) were counted after 3 days of incubation by 2 inde- 


\section{A VEGF}

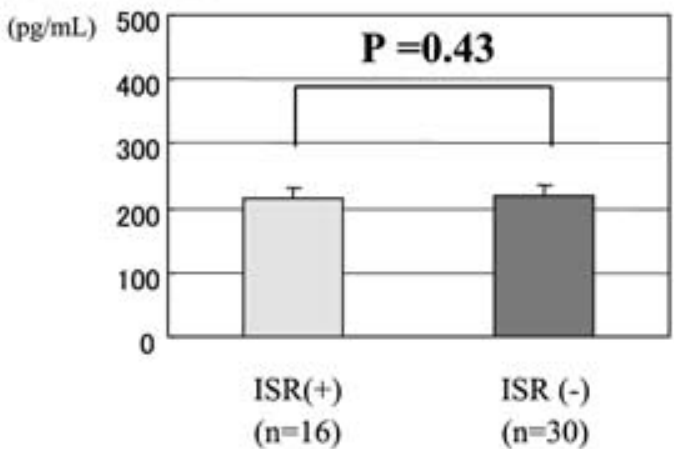

\section{HGF}

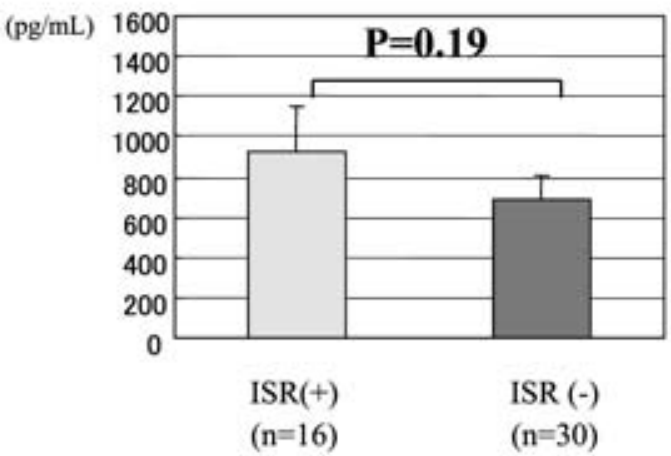

B b-FGF

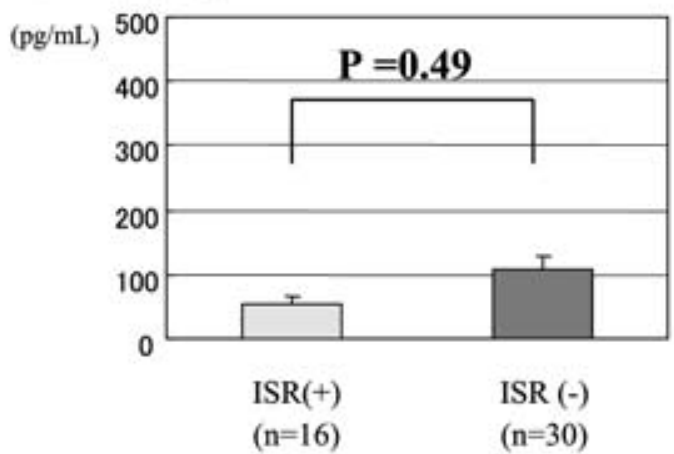

D $\quad$ MCP-1

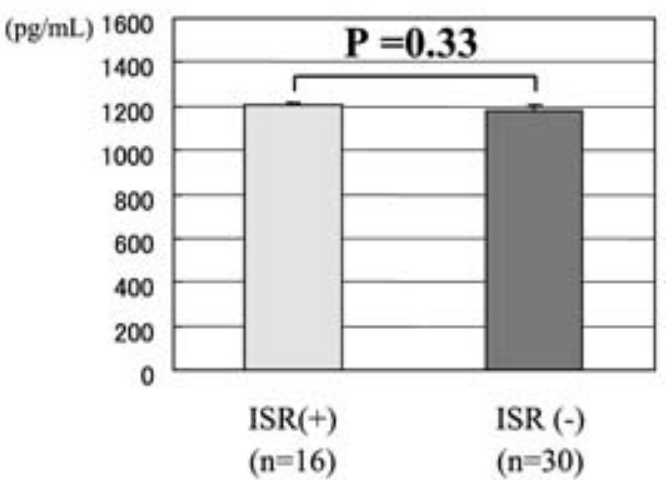

Figure 2. Secretion of angiogenic growth factors by EPCs over a 72-hour period. VEGF (A) indicates vascular endothelial growth factor; b-FGF (B), basic fibroblast growth factor; HGF (C), hepatocyte growth factors; MCP-1 (D), macrophage chemoattractant protein-1. ISR indicates instent restenosis.

pendent investigators. Conditioned media were separated and stored at $-80^{\circ} \mathrm{C}$ until measurement. They were assayed for the angiogenic growth factors, vascular endothelial growth factor (VEGF), basic-fibroblast growth factor (bFGF), hepatocyte growth factor (HGF), and chemokines, macrophage chemoattractant protein-1 (MCP-1) to assess the paracrine effects of EPCs. The angiogenic growth factors and chemokines were assayed by enzyme-linked immunosorbent assay (R\&D Systems, Inc., Minneapolis, MN, USA).

\section{Statistical analysis}

Data are expressed as means \pm SEM. Comparisons between two groups were analyzed by unpaired t test (two-sided). Correlations were assessed by Spearman's test. Independent predictors of in-stent restenosis were calculated by logistic regression. The following parameters were evaluated first in a univariate model: age, smoking, hypertension, diabetes mellitus, hyperlipidemia, family history, medications, type of lesion (A/B1 versus B2/C, according to the American College of Cardiology/American Heart Association classification), stent diameter, stented segment length, CFUs of EPCs, and senescence of EPCs defined as the percentage of $\beta$ galactosidase-positive cells for DAPI-positive cells. Variables with a probability value $<0.15$ were then entered into a mul- tivariate logistic regression analysis. All tests were done with Stat View J 5.0 (SAS Institute Inc., Cary, NC, USA). A value of $\mathrm{p}<0.05$ was considered statistically significant.

\section{Results}

\section{Patient characteristics and clinical data}

This study population contained patients suffering from acute coronary syndrome $(87.5 \%$ in patients with ISR and $86.7 \%$ without ISR). The frequency of the use of ACEI/ ARB (angiotensin-converting emzyme inhibitors /angiotensin receptor blockers) was higher and implanted stent diameter was smaller in patients with ISR. No drug-eluting stent was implanted in these subjects. No statistically significant differences existed between the two groups in terms of other clinical and angiographical characteristics (Table 1).

\section{Growth factor measurement}

To assess the paracrine effects of EPCs, we evaluated the arteriogenic cytokines secreted by EPCs. Over a 72-hour period, measurable amounts of VEGF, b-FGF, HGF and MCP1 were identified to be secreted by EPCs in both groups. However, No significant difference was found between two 

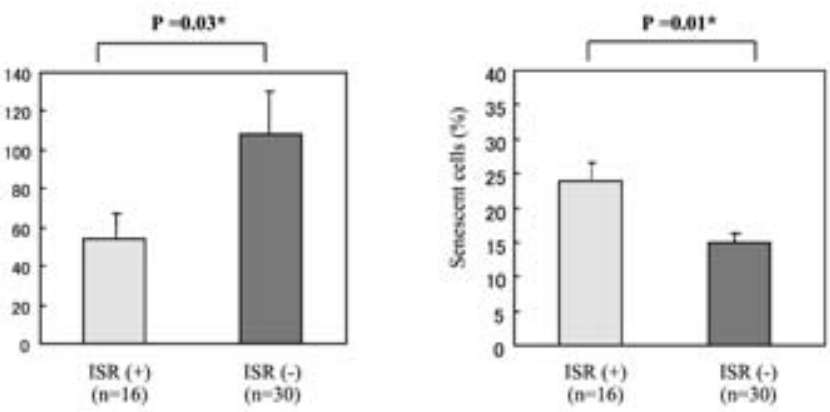

Figure 3. Functional activity of EPCs between the two groups (patients with and without in-stent intimal thickening). (A) Number of colony-forming units of circulating EPCs and (B) senescence-associated $\beta$-galactosidase activity ( $\beta$-gal). ISR indicates in-stent restenosis.

Table 2. Results of Univariate Logistic Regression Analysis according to Risk of In-stent Restenosis

\begin{tabular}{lccc}
\hline Variable & OR & $95 \% \mathrm{Cl}$ & $\mathrm{P}$ \\
\hline Age & 1.02 & $0.95-1.10$ & 0.56 \\
Family history & 0.73 & $0.18-2.87$ & 0.65 \\
Hypertension & 1.46 & $0.32-6.53$ & 0.62 \\
Diabetes Mellitus & 1.20 & $0.29-4.96$ & 0.81 \\
Hyperlipidemia & 1.59 & $0.41-6.23$ & 0.50 \\
Smoking & 1.46 & $0.32-6.53$ & 0.62 \\
$\beta$-bloker & 0.44 & $0.12-1.55$ & 0.20 \\
ACEls/ARBs & 3.76 & $0.71-19.9$ & 0.12 \\
Statins & 1.16 & $0.31-4.27$ & 0.83 \\
Ca antagonist & 0.85 & $0.21-3.39$ & 0.82 \\
Nitrates & 1.33 & $0.37-4.81$ & 0.66 \\
Nicorandil & 0.56 & $0.09-2.80$ & 0.43 \\
Lesion type B2/C & 2.32 & $0.53-10.0$ & 0.26 \\
Stent diameter & 0.14 & $0.02-0.92$ & 0.04 \\
Total stent length & 0.89 & $0.77-1.04$ & 0.15 \\
Colony-forming units of EPCs & 1.00 & $0.99-1.01$ & 0.55 \\
Senescence of EPCs & 1.06 & $1.00-1.13$ & 0.05 \\
\hline
\end{tabular}

ACEls indicates angiotensin-converting etcyme inhibitors: ARBs, angiotensin receptor blockers; EPCs, endoethelial peogenitor cells. Seneseence of EPCs was defined as the pereentage of $B$-zalactosidase-positive cells for DAPI-positive cells.

subjects (Fig. 2)

\section{Colony-forming units and senescence of EPCs}

To examine the functional activities of EPCs, we assessed the colony formation of EPCs. The number of CFUs was less abundant in patients with ISR than in those without ISR $(54 \pm 13$ vs. $113 \pm 22, p=0.03)$. In contrast to patients without ISR, patients with ISR had a greater number of senescent cells $(24 \pm 3$ vs. $15 \pm 1, \mathrm{p}=0.01)$ (Fig. 3).

At univariate logistic regression analysis (Table 2), the number of CFUs was not independently associated with ISR (OR $1.00,95 \%$ CI 0.99 to 1.01 ). At multiple logistic regression analysis (Table 3), increased number of senescent EPCs was the only variable associated with ISR (OR 1.10, 95\% CI 1.10 to 1.20$)$.

The potential influence of risk factors on CFUs and the
Table 3. Results of Multivariate Logistic Regression Analysis according to Risk of In-stent Restenosis

\begin{tabular}{llll}
\hline Variable & OR & $95 \%$ Cl & P \\
\hline ACEIs/ARBs & 3.35 & $0.42-26.9$ & 0.26 \\
Stent diameter & 0.18 & $0.02-1.84$ & 0.15 \\
Stent segment length & 0.86 & $0.72-1.02$ & 0.08 \\
Senescence of E.PCs & 1.10 & $1.01-1.20$ & 0.03 * \\
& & \\
\hline \\
OR indicates odds ratio; Cl, confidence interval; ACEls, angiotensin-converting encyme \\
inhibitors; ARBs, angiotensin receptor blockers; EPCs, endothelial progenitor cells. \\
"P-c0.05 is considered to be significant.
\end{tabular}

senescence of EPCs was evaluated. The number of risk factors was inversely correlated with the number of CFUs $(\mathrm{R}=-0.34, \mathrm{p}=0.02$ ) (Fig. 4A). In addition, the number of risk factors was correlated with the percentage of senescent cells $(\mathrm{R}=0.33, \mathrm{p}=0.03)$ (Fig. 4B).

\section{Discussion}

The present study showed that the senescence of EPCs was associated with ISR after coronary stenting in native coronary arteries. The association was independent of clinical data and angiographical variables known to be related to ISR. Therefore, the senescence, one of the functional characteristics of EPCs, might be involved in the pathophysiological process of ISR.

Endothelial denudation is considered to be a primary injury event after stent implantation. Endothelial cells (ECs) produce a significant number of basement membrane components and synthesize several growth factors and maintain the mitogenic quiescence of smooth muscle cell by the growth-inhibitory effect of nitric oxide (NO) (7). Therefore, promoting healing of the injured endothelium was the treatment and prevention of atherosclerosis and ISR. Several lines of evidence indicate that circulating EPCs can accelerate the reendothelialization process, such as the endothelialization in dog thoracic aorta Dacron graft (8), the recruitment of the surface of left ventricular-assist devices in humans (9), and homing to denuded parts of the artery after balloon injury (10).

A negative correlation between the number of CFUs and the number of coronary risk factors was demonstrated in this study, previously shown by Vasa $M$ et al (3). In addition, a positive correlation between the percentage of senescent EPCs and the number of coronary risk factors was demonstrated. This finding may support previous data by Hill et al (11), who showed that EPCs from subjects at high risk for cardiovascular events had higher rates of in vivo senescence than cells from subjects at low risk. Previous data showed that senescent EPCs induced by ex vivo cultivation (12), oxidized low-density lipoprotein (13) and angiotensin II (14), had a limiting capacity of proliferation and tube formation. These results suggest that the impairment of EPCs incorporation into neovascularization might lead to in-stent restenosis. 
(A)

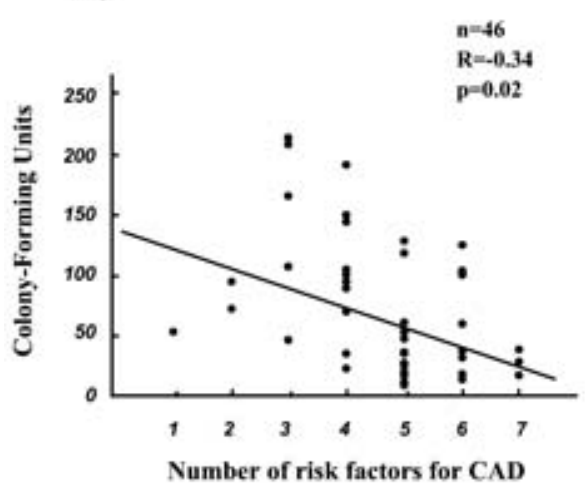

(B)

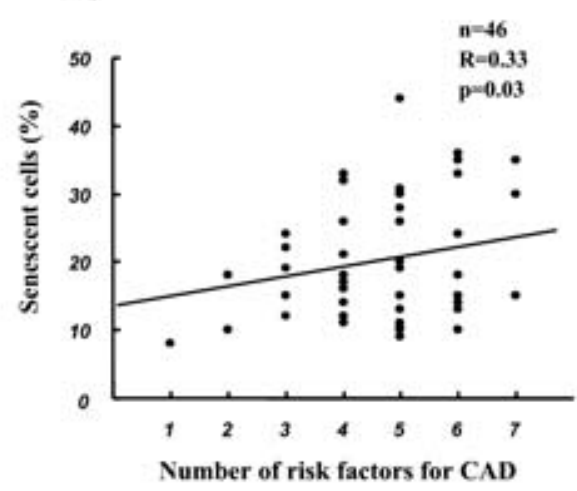

Figure 4. (A) The number of colony-forming units was inversely correlated with the number of risk factors for coronary artery disease (CAD). (B) The percentage of senescent cells ( $\beta$ galactosidase-positive cells/DAPI-positive cells) correlated with the number of risk factors for CAD.

Although the number of CFUs in patients without ISR was greater than that in patients with ISR, the number of CFUs was not an independent factor of ISR by multiple logistic regressions. It may be a mere reflection of other atherogenic risk factors in this clinical setting.

We evaluated the several growth factors (VEGF, HGF, and b-FGF) secreted from EPCs which might contribute to the endothelial proliferation. We didn't observe any significant differences between patients with and without ISR. However, endothelial cells release other molecules with antiinflammatory, antithrombotic, and antiproliferative effects which are crucial for the prevention of neointimal thickening. In addition, dysfunctional endothelium may release several substances, such as endothelin-1, angiotensin II, and growth factors and induce or enhance migration of smooth muscle cells and intimal hyperplasia, contributing to the process of ISR (15). Further study was needed in terms of paracrine effects that we could not investigate, contributing to the repairing process at the site of coronary stenting.

In the interpretation of our findings, several limitations must be considered.

First, we assessed the characteristics of EPCs of individual patients at 6 month after stent implantation. This study population contained patients suffering from acute myocardial infarction. Consequently, the number of EPCs at the time of stent implantation during recanalization was expected to be considerably affected under myocardial ische- mia and secreted cytokines (16). On the other hand, timecourse analysis of stent endothelialization disclosed nearcomplete endothelialization at 28 days after stent implantation in a rabbit model (17). The extent to which the characteristics of EPCs obtained 6 months after stenting represents those obtained just after stent implantation remains to be determined.

Second, we could not observe the destiny of circulating EPCs in vivo. It is actually unknown whether EPCs might participate in reendothelialization by homing to denuded parts of the injured artery, or by paracrine effects of secreting angiogenic growth factors. Third, the sample size was relatively small in our study in order to determine the causative mechanism of ISR. Further investigation was needed to identify the mechanism of contributory process of EPCs on in-stent restenosis.

\section{Conclusion}

This investigation shows the relationships between the senescence of EPCs and in-stent intimal thickening. The senescence of peripheral EPCs might imply potential contribution to the pathogenesis of ISR. The decreased functional capacity of EPCs might be a surrogate marker in the development of ISR after coronary stenting. This study leads to a reasonable understanding of the underlying mechanisms of ISR.

\section{References}

1. Bauters C, Isner JM. The biology of restenosis. Prog Cardiovasc Dis 40 (2): 107-116, 1997.

2. Asahara T, Murohara T, Sullivan A, et al. Isolation of putative progenitor endothelial cells for angiogenesis. Science 275: 964967, 1997.

3. Vasa M, Fichtlscherer S, Aicher A, et al. Number and migratory activity of circulating endothelial progenitor cells inversely correlate with risk factors for coronary artery disease. Circ Res 89: E17, 2001.
4. Werner N, Junk S, Laufs U, et al. Intravenous transfusion of endothelial progenitor cells reduces neointima formation after vascular injury. Circ Res 93: e17-24, 2003.

5. Vita JA, Treasure CB, Nabel EG, McLenachan JM, Fish RD, Yeung $\mathrm{AC}$, et al. Coronary vasomotor response to acetylcholine relates to risk factors for coronary artery disease. Circulation 81: 491-497, 1990.

6. Dimri GP, Lee X, Basile G, Acosta M, et al. A biomarker that identifies senescent human cells in culture and in aging skin in 
vivo. Proc Natl Acad Sci USA 92: 9363-9367, 1995.

7. Kipshidze N, Dangas G, Tsapenko M, et al. Role of the endothelium in modulating neointimal formation: vasculoprotective approaches to attenuate restenosis after percutaneous coronary interventions. J Am Coll Cardiol 44: 733-739, 2004.

8. Shi $\mathrm{Q}$, Rafii $\mathrm{S}, \mathrm{Wu} \mathrm{MH}$, et al. Evidence for circulating bone marrow-derived endothelial cells. Blood 92: 362-367, 1998.

9. Rafii S, Oz MC, Seldomridge JA, et al. Characterization of hematopoietic cells arising on the textured surface of left ventricular assist devices. Ann Thorac Surg 60 (6): 1627-1632, 1995.

10. Walter DH, Fichtlscherer S, Britten MB, et al. Statin therapy accelerates reendothelialization: a novel effect involving mobilization and incorporation of bone marrow-derived endothelial progenitor cells. Circulation 105: 3017-3024, 2002.

11. Hill JM, Zalos G, Halcox JP, et al. Circulating endothelial progenitor cells, vascular function, and cardiovascular risk. N Engl J Med 348: 593-600, 2003.

12. Assmus B, Urbich C, Aicher A, et al. HMG-CoA reductase inhibitors reduce senescence and increase proliferation of endothelial progenitor cells via regulation of cell cycle regulatory genes. Circ Res 92 (9): 1049-1055, 2003.

13. Imanishi T, Hano T, Sawamura T, Nishio I. Oxidized low-density lipoprotein induces endothelial progenitor cell senescence, leading to cellular dysfunction. Clin Exp Pharmacol Physiol 31: 407-413, 2004.

14. Imanishi $T$, Hano T, Nishio I. Angiotensin II accelerates endothelial progenitor cell senescence through induction of oxidative stress. J Hypertens 23: 97-104, 2005.

15. Patti G, Pasceri V, Melfi R, et al. Impaired flow-mediated dilation and risk of restenosis in patients undergoing coronary stent implantation. Circulation 111: 70-75, 2005.

16. Shintani S, Murohara T, Ikeda H, et al. Mobilization of endothelial progenitor cells in patients with acute myocardial infarction. Circulation 103: 2776-2779, 2001.

17. Asahara T, Chen D, Tsurumi Y, et al. Accelerated restitution of endothelial integrity and endothelium-dependent function after phVEGF165 gene transfer. Circulation 94: 3291-3302, 1996.

(C) 2006 The Japanese Society of Internal Medicine http://www.naika.or.jp/imindex.html 